\title{
PECTUS EXCAVATUM: INCREASE OF RIGHT VENTRICULAR SYSTOLIC, DIASTOLIC, AND STROKE VOLUMES AFTER SURGICAL REPAIR
}

Janusz Kowalewski, MD

Marian Brocki, MD, PhD ${ }^{\mathrm{a}}$

Tadeusz Dryjanski, MD ${ }^{\mathrm{b}}$

Krystian Zolyński, MD, $\mathrm{PhD}^{\mathrm{c}}$

Robert Koktysz, MD
Objective: The study was undertaken to assess how a surgical correction of funnel chest modifies right ventricular structure and function. Methods: Before and 6 months after surgery in 42 patients (27 male and 15 female patients, aged 5-31 years), a pectus index was calculated and echocardiographic examinations of the right ventricle were performed, with calculation of systolic, diastolic, and stroke volume indices. Right ventricular volume was estimated by subtracting the left ventricular volume from that of the entire heart. The values of the right ventricular volumes and the pectus index before and after the operation, as well as the changes in the indices, were compared. Results: Statistically significant changes in the pectus index and the right ventricular volume indices after surgery were noted. No correlation was observed between the changes in the pectus index and the changes in any right ventricular volume indices. Conclusion: Surgical treatment of funnel chest causes an increase in right ventricular systolic, diastolic, and stroke volumes, although there is no correlation between these changes and the degree of sternocostal elevation. (J Thorac Cardiovasc Surg 1999;118:87-93)
P atients with funnel chest have many anatomic, physiologic, genetic, biomechanical, and psychologic characteristics. ${ }^{1}$ Surgical correction of this deformity may be done for psychosocial reasons alone. ${ }^{2}$ Malfunction of the cardiovascular system in these patients is often called into question. ${ }^{3-7}$ It is difficult to prove, especially if the deformity is not severe. ${ }^{5,8-10}$

Although pectus excavatum is thought to impair mostly right ventricular performance, ${ }^{9}$ the degree of right ventricular dysfunction, if any, produced by this chest wall deformity remains controversial. ${ }^{5,11}$ The subjective physical improvement after the operation usually is not explained by changes in cardiorespiratory function. ${ }^{5}$ The purpose of this study was to determine how a sternocostal elevation in patients with funnel chest modifies right ventricular structure and function.

From The First Department of General Surgery, ${ }^{a}$ Department of Cardiology, ${ }^{\mathrm{b}}$ Department of Orthopedic and Field Surgery, ${ }^{\mathrm{c}}$ and Department of Patomorphology, ${ }^{\mathrm{d}}$ The Military Medical Academy, Lodz, Poland.

Received for publication April 29, 1997; revisions requested June 23, 1997; revisions received March 8, 1999; accepted for publication March 8, 1999.

Address for reprints: Janusz Kowalewski, MD, ul. Lisciasta 52/20, 91-357 Lodz, Poland.

Copyright $(\subseteq) 1999$ by Mosby, Inc.

$0022-5223 / 99 \$ 8.00+0 \quad \mathbf{1 2 / 1 / 9 8 4 2 3}$

\section{Patients and methods}

Study group. Between November 1987 and January 1996, 60 patients were operated on for funnel chest. There were 43 male and 17 female patients, and their ages ranged from 4 to 35 years. Before and 6 months after the operation, the patients underwent a complete physical examination, a chest radiograph in 2 views, an electrocardiogram, and a 2-dimensional echocardiogram.

Chest radiography. To determine the severity of the deformity in all the patients, we obtained the following measurements from the chest radiographs: (1) the minimal sternovertebral distance (the distance from the posterior border of the sternum to the anterior surface of the vertebral body on the lateral radiograph) and (2) the transverse diameter of the thorax, measured at the same level on the posteroanterior radiograph. ${ }^{12}$ The severity of pectus excavatum deformity, as well as the degree of sternocostal elevation resulting from the operation, were assessed in terms of a transverse thoracic dimension to sternovertebral distance. ${ }^{11}$ The larger the pectus index, the more severe the pectus deformity. The deformity was considered mild if the pectus index was less than 3.5; moderate, 3.5 to 4.5 ; and severe, more than 4.5. Patients with a pectus index less than 3.25 do not require surgical correction. ${ }^{11}$

The indications for operation were based on 2 principles: First, we operated any time we recognized significant deformity ${ }^{13}$; second, we were very reluctant to operate on a patient who did not ask for the operation. ${ }^{6}$

Surgical technique. The operation was performed with the patient under general anesthesia. During the operation, abnormal costal cartilages in the parasternal region were excised 


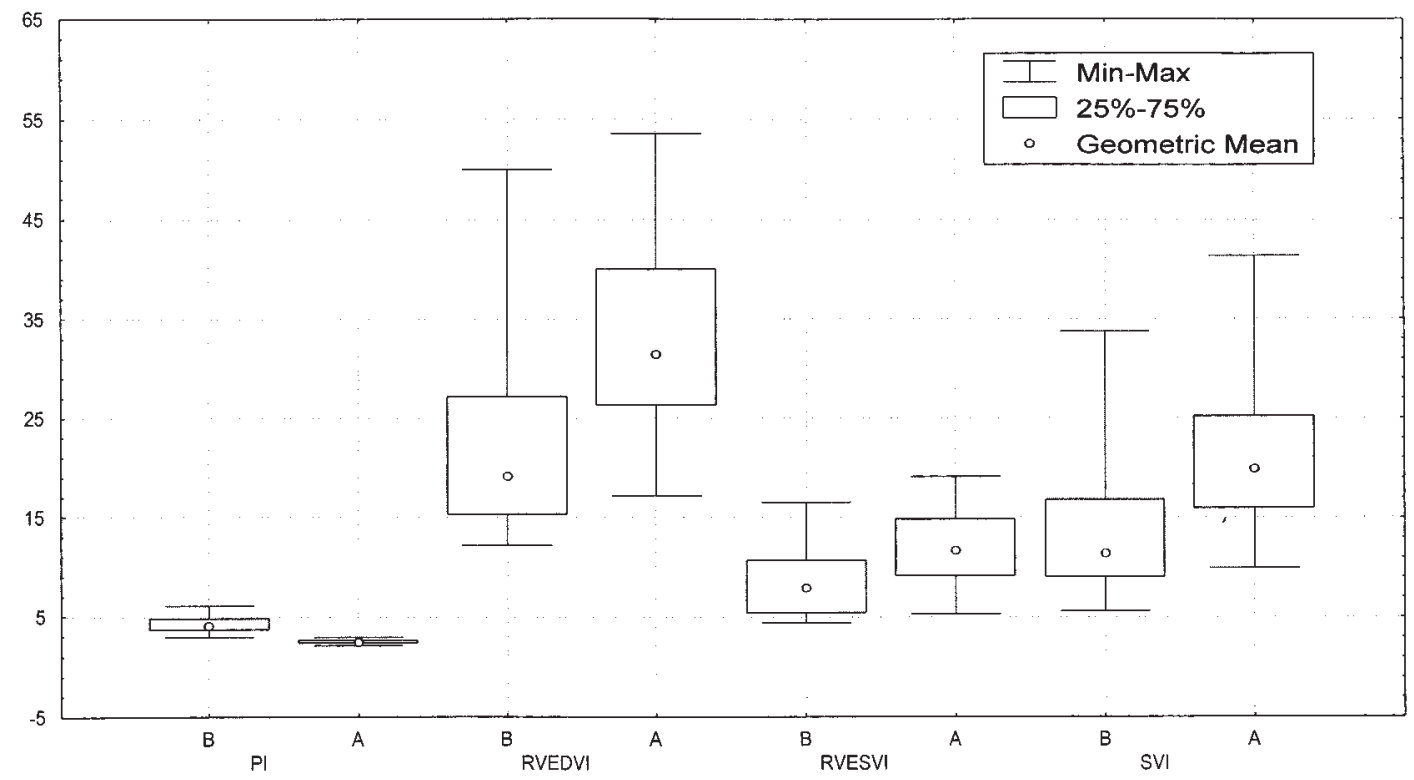

Fig 1. The "box and whiskers" presentation of pectus index $(P I)$, right ventricular end-diastolic (RVEDVI), end-systolic $(R V E S V I)$, and stroke $(S V I)$ volume indices before $(B)$ and after $(A)$ surgery. The points indicate the geometric means, the boxes encompass the first and the third quartiles, and the whiskers encompass the range of the values.

subperichondrially and bilaterally, and the sternum was cut transversely above the deformity and longitudinally across it. After that, the anterior thoracic wall was stabilized in the proper position with Kirschner wires.

Echocardiography. Before and 6 months after the operation, in 42 patients (27 male and 15 female patients, aged 531 years), echocardiographic examinations of the right ventricle were performed with calculation of systolic (RVESVI), diastolic (RVEDVI), and stroke (SVI) volume indices. The examinations were performed with the patient in the supine position. Right ventricular volume was calculated by subtracting the entire left ventricular volume (including the left ventricular myocardium) from the entire heart (including the right ventricle and the entire left ventricle). Both volumes (those of the entire heart and the entire left ventricle) were calculated as hemi-ellipsoid by the area-length method with the use of apical 4- and 2-chamber views. These examinations were standardized. In all cases, 5 cardiac cycles were estimated by 2 cardiologists.

Data analysis. All results of the indices were expressed as geometric means. The paired Wilcoxon test was used to compare the values of the indices before and after the operation in each individual patient.

The regression and correlation analysis was used to compare the values of pectus index and measured variables: RVEDVI, RVESVI, and SVI before and after the operation in each individual. In particular, we wanted to know the following: (1) the results of the correlation analysis between the relative changes (D\%) of RVEDVI, RVESVI, and SVI after the operation and the relative changes of pectus index (the degree of the sternocostal elevation) and (2) the results of the regression analysis of the absolute preoperative RVEDVI and SVI against the absolute preoperative pectus index alone.

\section{Results}

Of the 42 patients, 14 (33.3\%) were free of symptoms, and $27(64.3 \%)$ were psychologically disturbed by the abnormal shape of the chest. Twenty-four patients $(57.1 \%)$ had exercise limitations and 16 (38.1\%) had electrocardiographic changes (incomplete right bundle block and right axis deviation).

Six months after the operation, 2 patients $(4.7 \%)$ were still psychologically disturbed and 3 patients (7.1\%) reported exercise limitation. The electrocardiographic abnormalities did not change after repair.

All the obtained results of the geometric means, the first and the third quartile (P25 and P75), and the range of pectus index, RVEDVI, RVESVI, and SVI before and after the operation are presented in Fig 1.

Pectus index. The geometric mean of pectus index in our patients before the operation was 4.19 ( $\mathrm{P} 25=3.57$; P75 $=4.88)$. In 8 patients $(19.0 \%)$ the deformity was mild, in $16(38.1 \%)$ moderate, and in $18(42.9 \%)$ severe. After the operation the geometric mean decreased to 2.47 (P25 = 2.33; P75 = 2.65). The index was significantly smaller after the operation $(P<.0001)$.

RVEDVI. The geometric mean of RVEDVI in our 


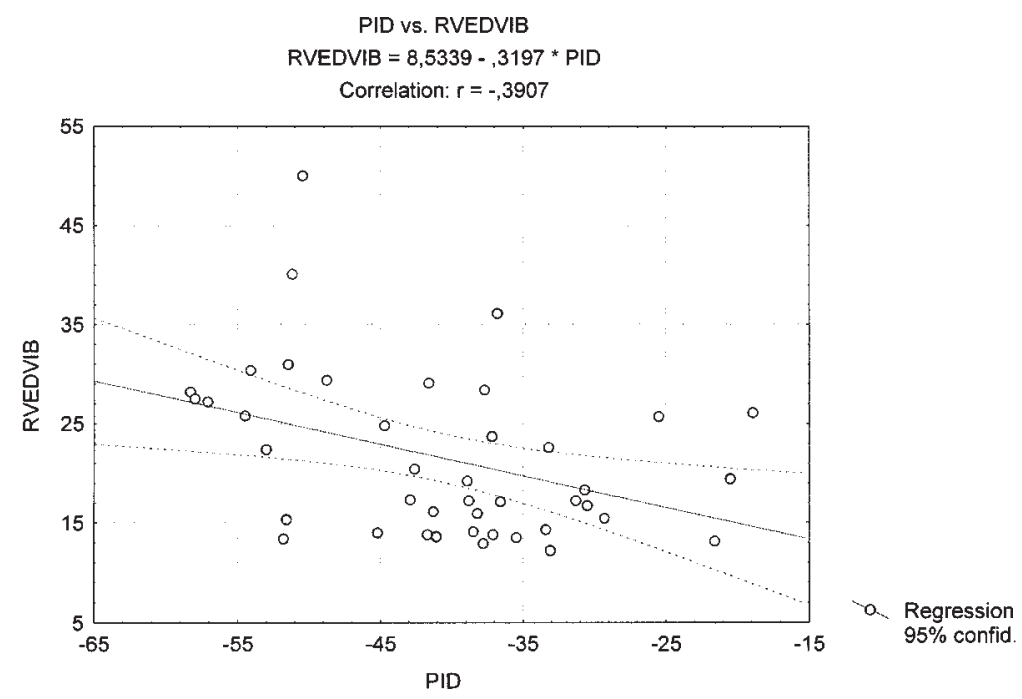

Fig 2. Linear regression analysis of the preoperative absolute RVEDVI against the relative change in pectus index. $P I D$, Pectus index difference; $R V E D V I B$, right ventricular end-diastolic volume index before the operation.

Table I. Correlation coefficients between pectus index (PI) and measured variables-RVEDVI, RVESVI, and SVIbefore $(B)$ and after $(A)$ surgery

\begin{tabular}{lrrrrrrrrr}
\hline & $R V E D V I B$ & RVEDVIA & RVEDVI $D$ & RVESVI $B$ & RVESVI $A$ & RVESVI $D$ & \multicolumn{1}{c}{ SVI $B$} & SVI A & SVI $D$ \\
\hline PI & -0.222 & -0.112 & 0.123 & -0.200 & -0.172 & 0.056 & -0.154 & -0.060 & 0.138 \\
B & $P=.158$ & $P=.479$ & $P=.439$ & $P=.204$ & $P=.275$ & $P=.723$ & $P=.331$ & $P=.706$ & $P=.384$ \\
PI & -0.040 & 0.141 & 0.234 & -0.066 & 0.019 & 0.113 & 0.023 & 0.252 & 0.295 \\
A & $P=.800$ & $P=.373$ & $P=.135$ & $P=.676$ & $P=.907$ & $P=.476$ & $P=.884$ & $P=.108$ & $P=.058$ \\
PI & -0.391 & -0.186 & 0.255 & -0.411 & -0.037 & 0.357 & -0.386 & -0.365 & 0.048 \\
D & $P=.011$ & $P=.238$ & $P=.103$ & $P=.007$ & $P=.815$ & $P=.070$ & $P=.012$ & $P=.068$ & $P=.762$ \\
\hline
\end{tabular}

$D$, Change in measured parameters.

patients before the operation was 20.15 (P25 = 15.12; $\mathrm{P} 75=27.06)$ and after the operation, $31.56(\mathrm{P} 25=$ 26.12; P75 = 40.42). The index increased significantly after the operation $(P<.0001)$.

RVESVI. The geometric mean of RVESVI before the operation was $7.86(\mathrm{P} 25=5.21 ; \mathrm{P} 75=10.72)$ and after surgical correction, $11.40(\mathrm{P} 25=9.03$; $\mathrm{P} 75=$ 14.91). Despite the fact that the index decreased after the operation in 6 patients, generally it was significantly higher $(P<.0001)$.

SVI. The geometric mean of right ventricular SVI in our patients before the operation was 11.82 (P25 = 8.93; P75 = 16.48) and after sternocostal elevation, $19.94($ P25 = 15.68; P75 = 25.17). SVI increased significantly after the operation $(P<.0001)$.

The results of regression and correlation analysis between pectus index and the measured variables, RVEDVI, RVESVI, and SVI, before and after the operation are presented in Table I.
A significant inverse correlation was observed between the relative change in pectus index after repair and the preoperative right ventricular volumes: RVEDVI B (before the operation) $(r=-0.39 ; P=.011)$, RVESVI B $(r=-0.41 ; P=.007)$, and SVI B $(r=-0.39$; $P=.012$ ) (Figs 2, 3, and 4).

No correlation was observed between the relative changes after the operation in the pectus index used and the changes in RVEDVI, RVESVI, or right ventricular SVI in particular individuals.

The results of the regression analysis of the absolute preoperative RVEDVI and SVI against the absolute pectus index (PI) are reported in the form of 2 regression equations:

$$
\text { RVEDVI }=-0.222 \mathrm{PI}+41.664
$$

$\mathrm{F}(1.40)=2.075 ; P<.157$; standard error of the estimate: $8.238 ; R^{2}=0.049$.

$$
\mathrm{SVI}=-0.154 \mathrm{PI}+22.352
$$




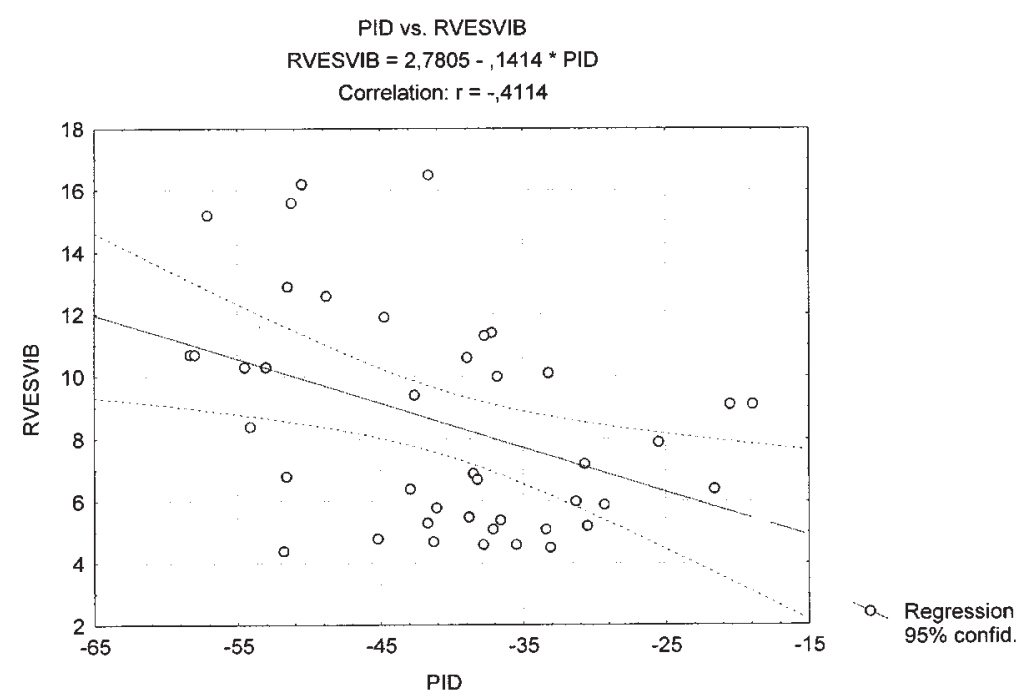

Fig 3. Linear regression analysis of the preoperative absolute RVESVI against the relative change in pectus index. $P I D$, Pectus index difference; RVESVIB, right ventricular end-systolic volume index before the operation.

$\mathrm{F}(1.40)=0.967 ; P<.331$; standard error of the estimate: $5.714 ; R^{2}=0.023$.

\section{Discussion}

The majority of our patients $(81 \%)$ had moderate or severe deformity of the chest, and more than half of them had exercise limitations. Surgery afforded them cosmetic and functional improvement. The pectus index used was significantly smaller after repair, and exercise tolerance in the patients increased.

Points of view about cardiac function in patients with funnel chest and about the influence of surgery on cardiac function are often contradictory.5,9,14 The lack of correlation between the severity of symptoms and the severity of anatomic or physiologic parameters is consistent with the majority of studies. ${ }^{1,2,9,15}$

Many authors believe that surgical treatment is indicated for cosmetic and psychologic reasons in most cases of funnel chest deformity., 4, 16,17 Most also believe that surgery has no physiologically significant effect on cardiac function and cardiorespiratory response to exercise. ${ }^{6-8}$ Others have proved that more than $90 \%$ of patients have improvement in exercise tolerance, endurance, and cardiac and respiratory symptoms after the operation, ${ }^{3}$ but this improvement is not explained by changes in cardiorespiratory function. ${ }^{3,7,8}$

Before the operation, the patient with pectus excavatum usually is not significantly different from normal subjects with respect to cardiac index, left ventricular ejection fraction, left ventricular end-diastolic volume index, end-systolic volume index, or stroke volume index at rest or during exercise..$^{918}$ Only the maximal exercise and immediate postexercise diastolic pressures are significantly elevated in the patients with pectus excavatum when compared with their matched control subjects. ${ }^{18}$ Aortic, left atrial, and left ventricular dimensions are similar to those of normal subjects. ${ }^{12}$

In this article we concentrated on the right ventricle, but a parallel study of left ventricular function was undertaken as well. ${ }^{19}$ The changes in left ventricular function after surgery seem to be more difficult to classify, and in our opinion more cases are needed to complete the study. In fact, SVI of the left ventricle increased significantly after the operation, but only in the patient subset with severe deformities. The sternocostal elevation did not change left ventricular function in patients with mild or moderate deformities.

The major differences in resting values compared with those in normal subjects are in terms of right ventricular function. ${ }^{9,12,20}$ The right ventricle is less concentric, more anterior in relation to the sternum, and more distensible than the left ventricle. ${ }^{9,15,20}$ Although right ventricular anatomy might be altered by sternal compression of the right atrium and right ventricle, ${ }^{9}$ there is no relation between right ventricular functional impairment and the degree of chest wall deformity. ${ }^{12}$ Cardiac catheterization studies ${ }^{14}$ disclosed pressure gradients between the right ventricle and the pulmonary artery, perhaps caused by compression of the pulmonary outflow tract by the sternum.

The results before surgery in our patients were not compared with the results in healthy individuals. 


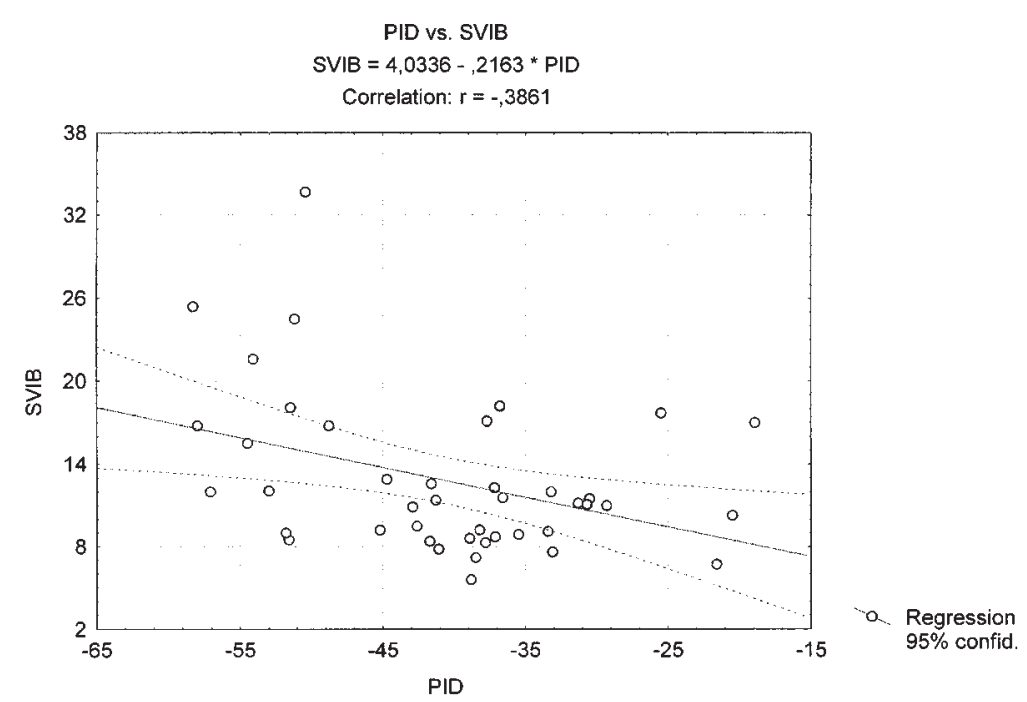

Fig 4. Linear regression analysis of the preoperative absolute SVI against the relative change in pectus index. $P I D$, Pectus index difference; SVIB, stroke volume index before the operation.

However, we defined the correlations between preoperative right ventricular volumes and the degree of the deformity. After pectus excavatum repair, RVEDVI, RVESVI, and SVI importantly increased, probably because of decreased sternal compression of the mediastinum. However, no correlation between the quantitative degree of pectus relief and the level of improvement in right ventricular function could be established. The possible explanation for the lack of the correlation is the fact that the degree of sternocostal elevation resulting from the operation is usually large in comparison with the degree of right ventricular decompression. In other words, the right ventricular volumes may increase in a linear fashion with the increase in thoracic diameter after repair until the decompression of the heart is completed (the presence of a threshold effect). After that point, the further elevation of the sternum improves the thoracic shape but has no influence on the improvement of right ventricular function.

A significant inverse correlation was detected between the preoperative right ventricular volumes and the relative change in pectus index after the operation: that is, the smaller the right ventricular volume before the repair, the greater the perioperative change in pectus index. Because the degree of sternocostal elevation resulting from a properly done operation is greater in patients with more severe deformity, this correlation confirms indirectly the fact that the greater impact on the right ventricle is made by the more concave sternum.

Despite the cosmetic improvement, the absolute RVESVI decreased after the operation in 6 of our patients. This patient subset consisted of 5 boys and 1 girl aged 14 to 19 years, and the deformities were classified as mild in 4 and moderate in 2 of them. The possible explanation for such results may be a lack of significant compression of the heart by the sternum before the operation. Surgical correction improved the thoracic shape in these patients, but it did not improve right ventricular function.

Our data support the speculations that factors other than changes in lung volumes are responsible for the subjective $^{8}$ and objective ${ }^{11}$ physical improvement after surgery in most patients.

Determination of right ventricular volume is conceptually more difficult than that of left ventricular volume. ${ }^{21-23}$ Whereas the left ventricle resembles an ellipsoid, a convenient volumetric model, the right ventricle has a complex and crescentic shape, defying simple geometric description. ${ }^{12,22}$

RVEDVI before pectus excavatum repair is described as significantly smaller than in normal subjects and, after surgery, becomes not significantly different from that of normal subjects. ${ }^{9}$

We are aware of the limitations of the echocardiographic subtraction method used in our clinical study. Usually larger right ventricular volumes are more underestimated than smaller volumes, ${ }^{22,23}$ because it generally appears to be technically more difficult to record the maximum area of the larger end-diastolic heart than that of the smaller end-systolic heart. ${ }^{23} \mathrm{~A}$ second possible explanation for underestimation of the larger volume may be related to underestimation of the right ventricu- 
lar outflow tract volume. ${ }^{21,22}$ This method does not take into account the outflow tract volume, although it appears to be larger in diastole than in systole. ${ }^{23}$

We conclude that surgical repair of funnel chest causes a significant increase of RVEDVI, RVESVI, and SVI; however, there is no correlation between the improvement of right ventricular structure and function and the degree of sternocostal elevation.

\section{REFERENCES}

1. Haller JA. Operative management of chest wall deformities in children: unique contributions of southern thoracic surgeons. Ann Thorac Surg 1988;46:4-7.

2. Humphreys GH, Jaretzki A. Operative correction of pectus excavatum: late results with and without operation. J Thorac Cardiovasc Surg 1980;80:686-95.

3. Fonkalsrud EW, Salman T, Guo W, Gregg JP. Repair of pectus deformities with sternal support. J Thorac Cardiovasc Surg 1994; 107:37-42.

4. Goertzen M, Baltzer A, Schulitz KP. Long-term results after operation for funnel chest. Arch Orthop Trauma Surg 1993;112:289-91.

5. Morshuis WJ, Folgering HT, Barentsz JO, Cox AI, van-Lier HJ, Lacquet LK. Exercise cardiorespiratory function before and one year after operation for pectus excavatum. J Thorac Cardiovasc Surg 1994;107:1403-9.

6. Morshuis WJ, Mulder H, Wapperom G, Folgering HT, Assman M, Cox AL, et al. Pectus excavatum: a clinical study with longterm postoperative follow-up. Eur J Cardiothorac Surg 1992;6: 318-28.

7. Wynn SR, Driscoll DJ, Ostrom NK, Staats BA, O'Connel EJ, Mottram CD, et al. Exercise cardiorespiratory function in adolescents with pectus excavatum: observation before and after operation. J Thorac Cardiovasc Surg 1990;99:41-7.

8. Morshuis WJ, Folgering HT, Barentsz JO, van-Lier H, Lacquet LK. Pulmonary function before surgery for pectus excavatum and at long term follow-up. Chest 1994;105:1646-52.

9. Peterson RJ, Young G, Godwin JD. Noninvasive assessment of exercise cardiac function before and after pectus excavatum repair. J Thorac Cardiovasc Surg 1985;90:251-5.

10. Seliem MA, Duffy CE, Gidding SS. Echocardiographic evaluation of the aortic root and mitral valve in children and adolescents with isolated pectus excavatum: comparison with Marfan patients. Pediatr Cardiol 1992;13:20-3.

11. Quigley PM, Haller JA Jr, Jelus KL. Cardiorespiratory function before and after corrective surgery in pectus excavatum. J Pediatr 1996;128:638-43.

12. Mocchegiani R, Badano L, Lestuzzi C, Nicolosi GL, Zanuttini D. Relation of the right ventricular morphology and function in pectus excavatum to the severity of the chest wall deformity. Am J Cardiol 1995;76:941-6.

13. Haller AJ Jr, Scherer LR, Turner CS, Colombani PM. Evolving management of pectus excavatum based on a single institutional experience of 664 patients. Ann Surg 1989;209:578-83.

14. Beiser G.D., Epstein SE, Stampfer M. Impairment of cardiac function in patients with pectus excavatum with improvement after operative correction. N Engl J Med 1972;208:267-71.

15. Clausner A, Clausner G, Basche M, Blumentritt S, Layher F, Vogt L. Importance of morphological findings in the progress and treatment of chest wall deformities with special reference to the value of computed tomography, echocardiography and stereophotogrammetry. Eur J Pediatr Surg 1991;1:291-7.

16. Cahill JL, Less GM, Robertson TH. A summary of preoperative and postoperative cardiorespiratory performance in patients undergoing pectus excavatum and carinatum repair. J Pediatr Surg 1984;19:430-6.

17. Mansour KA, Anderson TM, Hester TR. Sternal resection and reconstruction. Ann Thorac Surg 1993;55:838-42.

18. Ghory M, James FW, Mays W. Cardiac performance in children with pectus excavatum. J Pediatr Surg 1989;24:751-5.

19. Kowalewski J, Barcikowski S, Brocki M. Cardiorespiratory function before and after operation for pectus excavatum: mediumterm results. Eur J Cardiothorac Surg 1998;13:275-9.

20. O'Callagham C, Champan B, Howseman A, Stehling M, Coxon R, Mansfield P. Echo planar imaging of an infant with pectus excavatum. Eur J Pediatr 1990;149:698-9.

21. Bommer W, Weinter L, Neumann A, Neef J, Mason DT, DeMaria A. Determination of right atrial and right ventricular size by twodimensional echocardiography. Circulation 1979;60:91-100.

22. Levine RA, Gibson TC, Aretz T. Echocardiographic measurement of right ventricular volume. Circulation 1984;3:497- 505.

23. Tomida M, Masuda H, Sumi T, Shiraki H, Gotoh K, Yagi Y, et al. Estimation of right ventricular volume by modified echocardiographic subtraction method. Am Heart J 1992;123:1011-22. 